\title{
Electroencephalographic Activities as Biomarker in the Accumulative Dose of Alcoholic Drinker: A Preliminary Study
}

\author{
Phakkharawat Sittiprapaporn \\ Brain Science and Engineering Innovation Research Unit \\ School of Anti-Aging and Regenerative Medicine \\ Mae Fah Luang University, Bangkok, Thailand \\ Email: wichian.sit@mfu.ac.th
}

\author{
Djohan \\ Graduate School of Indonesia Institute of the Arts \\ Indonesia Institute of the Arts \\ Yogyakarta, Indonesia \\ Email: djohan.djohan@yahoo.com
}

\begin{abstract}
The attentional processing of the human brain during the discrimination of supra-segmental features of Thai phonemes related to working memory was investigated. The electroencephalographic activities of accumulative dose of alcohol were investigated while the measurement of brain function of the cognitive task of discrimination of supra-segmental features of Thai phonemes. The cognitive effort caused changes by the difficulty in discriminating supra-segmental features of Thai phonemes were reflected in specific electroencephalographic signals. A 14-channels electroencephalogram (EMOTIV Inc., USA) was used to record the electrical activities. The electrode array was placed according to the international 10-20 system. Both earlobes were used as references. Electroencephalographic activities were recorded in two different periods; resting period and cognitive task. In the cognitive task, the participants were asked to perform the cognitive task in order to measure their brain function of discrimination of supra-segmental features of Thai phonemes. The SuperLab program were used to deliver all stimuli to the participants. The result showed that there was a neuronal population related to the working memory activating strongly at the right medial temporal gyrus.
\end{abstract}

Keywords-Acohol; Brain; Electroencephalogrm (EEG); Standardized Electromagnetic Tomography (sLORETA)

\section{INTRODUCTION}

The consumption of alcohol is generally known to effect on human brain function, cognition, and performance. It has been focused on the research attention and memory. Alcoholics have also reduced cortical grey and white matter volumes when compared to light drinkers. It is generally accepted that both white and grey matter damages in relation to functional connectivity between distinct brain regions may be disturbed in alcoholics. Generally, in the social situations, alcohol is usually consumed in the cumulative fashion. That means most of people consume a number of less potent drinks over of several

* Corresponding author: Assist. Prof. Phakkharawat Sittiprapaporn, Ph.D., Brain Science and Engineering Innovation Research Unit (BSEI), School of Anti-Aging and Regenerative Medicine (AARM), Mae Fah Luang University, Bangkok, Thailand; Email: wichian.sit@mfu.ac.th

\section{Roungsan Chaisricharoen}

Brain Science and Engineering Innovation Research Unit School of Information Technology

Mae Fah Luang University, Chiang Rai, Thailand

Email: roungsan.cha@mfu.ac.th

hours rather than drinking a single dose of alcohol in a period of 10-20 min [1]. These drinking behaviors have been reported in previous studies. These previous studies investigated the effects of alcohol on speech perception under realistic social drinking situation [1-2].

Theoretically, Event-related Potentials (ERPs) components are known to monitor the detection of consciousness with several features of sounds. These features include the physical feature, the semantic feature, and the syntactic deviation, respectively. The ERP recordings have been used in investigating the insight of the neuronal events especially in relation to the auditory change detection of the human brain function. The probing the neural processes preceding the involvement of the attentional mechanisms were done by the ERP recording [3]. The present study investigated the attention and memory process in the human brain while discriminating the cognitive tasks composing of the supra-segmental features of monosyllabic Thai words. In order to locate the multiple non-dipolar sources, the standardized low-resolution electromagnetic tomography (sLORETA) calculation were also applied.

\section{MATERIALS AND METHODS}

\section{A. Participants:}

The experiment was done with five participants, aged between 32-47 years old. All participants were in good health. No congenital illness, no record of brain surgery, not taking medicines or drugs that affect to nervous system were personally reported. All participants were reported that they regularly alcoholic drinker. The left handedness was set as the exclusion criteria. Moreover, other criteria include history of medical and neurologic diseases, psychiatric disorders, head trauma or assumption of central nervous system active drugs in the two weeks prior to study entry were strictly controlled. However, if we found the presence of EEG abnormalities at 
the baseline recording occurring during the measurement, the participants were excluded. Finally, the experimenter explained the purposes of the present study as well as other details before providing the informed consent to participants to sign in. The written consents were collected from all participants before participating in the study. The informed consents were managed regarding the regulation of Helsinki declaration.

\section{B. Procedures:}

Electroencephalographic activities effected by the accumulative does of alcohol was investigated with participants who personally reported as accumulative alcoholic drinkers. Four pairs of physically different suprasegmental features of monosyllabic Thai words were included in the cognitive task in which all participants kept doing. The cognitive task of two blocks, 50 trails per block, were randomly order to the participants to complete.

\section{Electroencephalographic recording:}

The 14 channels EEG using EMOTIV system (EMOTIV, Inc., USA) was used to record the electrical activities in the human brain. Regarding to the international 10-20 system of electrode placement, the electrode array was placed on the participants' heads. Both earlobes were used as the references. The bandpass filtered was set at $0.05-100 \mathrm{~Hz}$. They were also digitized at the $1,000 \mathrm{~Hz}$ in order to process the EEG signals. The average frequency domains were obtained from those presented stimuli. EEG activity was recorded during the measurement of accumulative alcohol drinkers' brain function in the different supra-segmental features of monosyllabic Thai words discrimination. SuperLab software (Cedrus Corporation, San Pedro, USA) was used to deliver all stimuli to the participants during the experiment (see Table 1.)

Table I. The discrimination of stimuli presented by the stimulus presentation

\begin{tabular}{lll}
\hline Stimuli & & Supra-Segmental Features of Monosyllabic Thai Words \\
\hline$\square^{\mathrm{H}}$ & $\square^{\mathrm{L}}$ & same short vowel, different pitch \\
$\square \square^{\mathrm{L}}$ & $\square \square^{\mathrm{R}}$ & same long vowel, different pitch \\
\hline
\end{tabular}

Remak : $\square$ represents short vowel; $\square \square$ represents long vowel; $\square^{\mathrm{H}}$ represents short vowel with high pitch; $\square^{\mathrm{L}}$ represents short vowel with low pitch; $\square \square^{\mathrm{L}}$ represents long vowel with low pitch; $\square \square^{\mathrm{R}}$ represents long vowel with rising pitch.

\section{Computation of power spectra:}

sLORETA imaging program [11] was used to analyze and display the three-dimensional distribution of the cortical generators of all participants' brain. According to PascualMarqui et al. [11], sLORETA calculates the current source density distribution in the human brain. These current source density distributions contribute to the electrical scalp field at each of 2395 voxels in the gray matter and the hippocampus. These references brains are adopted from the MNI 305, Brain Imaging Centre, Montreal Neurological Institute. Importantly, the sLORETA calculated the three-dimensional source generator of the electrical activities. These sources of electrical activities were contributed to the electrical scalp field of all participants. These contributions were defined by the regions of interest (ROI) on the basis of local maxima of the sLORETA distribution [11-12]. It is based on the linear weighted sum of the scalp electric potentials [11]. Additionally, the applied version of sLORETA used a threeshell spherical head model aligned to the Talairach space. Based on SLORETA software, the sLORETA will choose the smoothest of all possible current density configurations throughout the human brain volume. The sLORETA will then maximize the total squared Laplacian of source strengths. However, the implication of the sLORETA method is only shown that all neighboring voxels have a maximally similar electrical activity [11-12].

\section{E. Statistical analysis:}

After logarithmic transformation, paired t-test statistics was used to compared between sLORETA images showing information of the cortical sources. Statistical tests at $p \leq 0.05$ (two-tailed) were set as significance level.

\section{RESULTS AND DISCUSSOIN}

EEG signals reflected by the discrimination of all stimuli revealed changes in the cognitive effort using the suprasegmental features of monosyllabic Thai words. The threedimensional distribution was calculated by sLORETA. The representation of the cortical generators was also analyzed. It revealed the changes of difficulty in discriminating suprasegmental features of monosyllabic Thai words. These changes showed strongly activated in the right medial temporal gyrus (70, -20, -10; BA21) (see fig. 1-4).

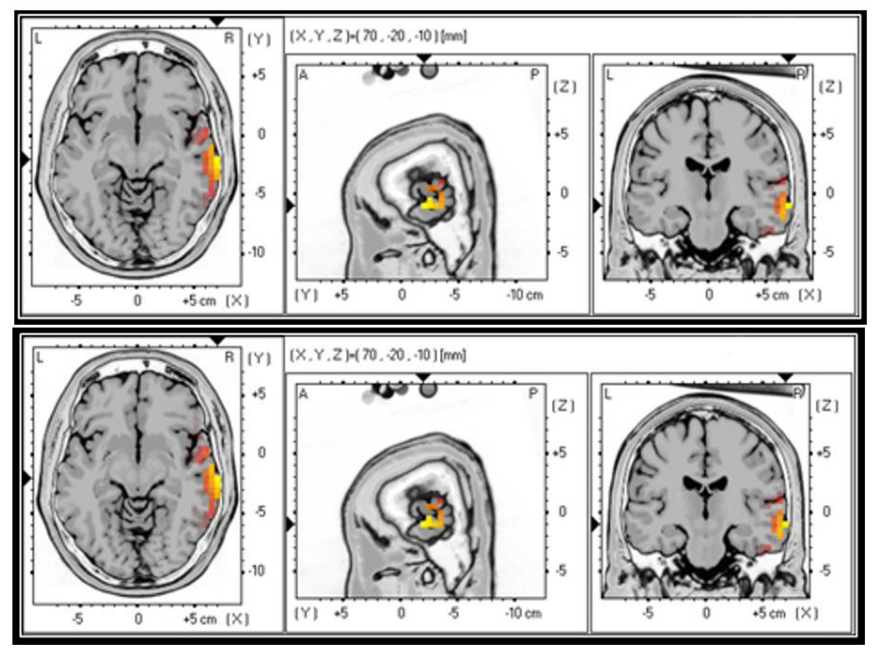

Fig.1 sLORETA was used to calculate the xyz-values in Talairach space of the frequency domain window. The discrimination of stimuli showed strongly activated in the right medial temporal gyrus (MTG) $(70,-20,-10$; BA21) for $/ \square^{\mathrm{H} /}(\mathrm{Top} ; 5.16 \mu \mathrm{V})$ and $/ \square^{\mathrm{L}} /($ Bottom; $4.83 \mu \mathrm{V})$, respectively. 


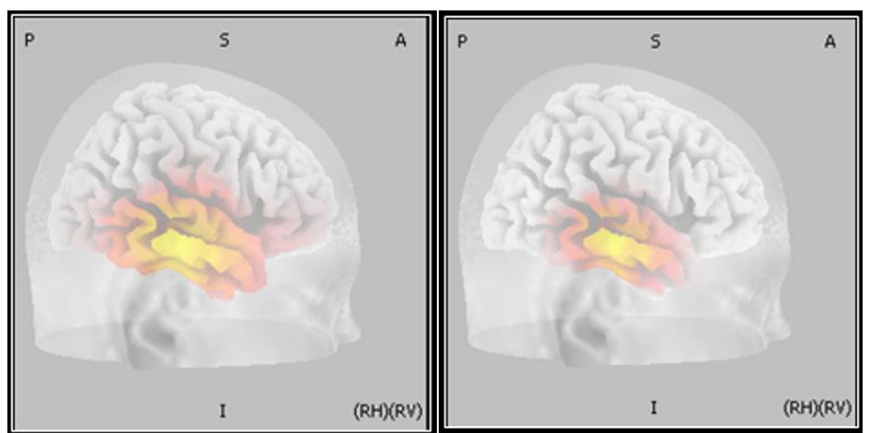

Fig. 2 sLORETA was used to calculate the source generator of the frequency domain window. The source generator of changes for $/ \square^{\mathrm{H}} /(\mathrm{Left})$ and $/ \square^{\mathrm{L}} /$ (Right) activated more strongly the right hemisphere (RH) (see description in Fig. 1).

The attention and memory processes in the human brain were previously reported that they were related to changes of neural representation in the human brain. These changes of neuronal representation were induced by new sensory inputs [12-17]. In the present study, the discrimination ability reflected by the cognitive task composing of the suprasegmental features of monosyllabic Thai words was also reflected in the attention and memory processes. These processes were revealed by the power spectra of EEG activities. The electrophysiological activities in temporal areas in this study are stayed in the line with other previous studies [18-19]. Several previous studies have been characterized the human brain responses to different sets of novel stimuli including the identifiable and non-identifiable sounds. In addition, these previous studies also demonstrated the activation of fronto-parietal areas and temporal network of the human brain responses [18-19].

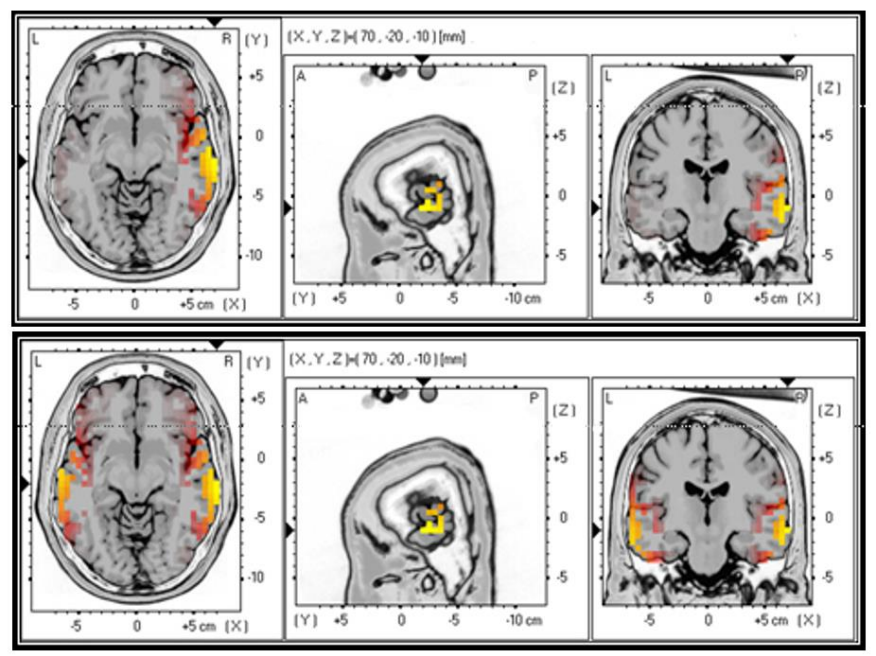

Fig. 3 sLORETA was used to calculate the xyz-values in Talairach space of the frequency domain window. The discrimination of stimuli showed strongly activated in the right medial temporal gyrus (MTG) $(70,-20,-10$; BA21) for $/ \square \square^{\mathrm{L}} /(\mathrm{Top} ; 1.76 \mu \mathrm{V})$ and $/ \square \square^{\mathrm{R}} /($ Bottom; $2.18 \mu \mathrm{V})$, respectively.

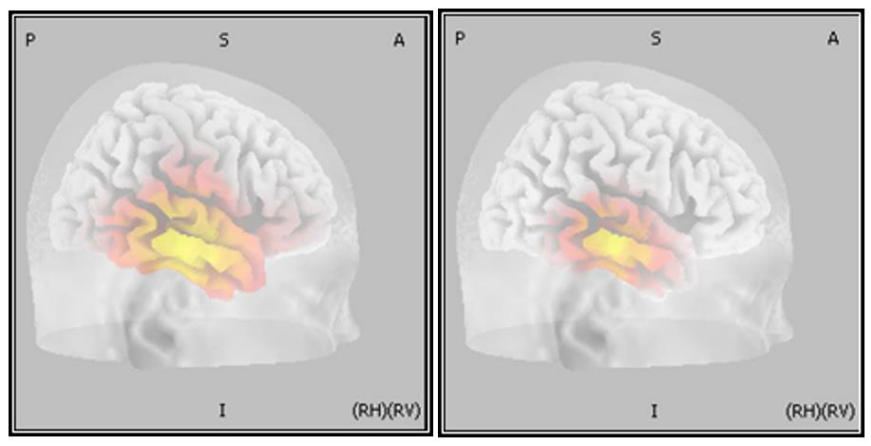

Fig. 4 sLORETA was used to calculate the source generator of the frequency domain window. The source generator of changes for $/ \square \square^{\mathrm{L}} /(\mathrm{Left})$ to $/ \square \square^{\mathrm{R}} /$ (Right) activated more strongly the right hemisphere (RH) (see more description in Fig. 3)

According to the sLORETA methodology, the sLORETA source imaging restricts the localization of electrical generators to cortical gray matter and hippocampus naming the technically "blurred" spatial resolution [11-12]. Although the exclusion of the involvement of subcortical structures in the sLORETA solution space, the possible involvement of subcortical structures from the sLORETA solution space were also investigated with the power spectra [19].

\section{CONCLUSION}

The present study measured the electroencephalographic activities in the accumulative alcoholic drinkers' brain while processing the cognitive tasks. The present study used the supra-segmental features of monosyllabic Thai words discrimination as the cognitive tasks. The result showed that it reflected in specific electroencephalographic signals in the accumulative alcoholic drinkers' brain. The neuronal populations might need for accumulative alcoholic drinkers' working memory processing in the brain. Additionally, the processing was taken place in the right medial temporal gyrus accessed by the sLORETA.

\section{ACKNOWLEDGMENT}

This research was fully supported by grant no. 61210320031 (31/2018) under the "Electroencephalographic Studies of Biomarker in the Human Brain of Alcohol Dependence Syndrome during the Processing of Informative Perception Project" funding by Mae Fah Luang University, Thailand. We thank all of the participants involved in this preliminary study as main data provider of this study.

\section{REFERENCES}

[1] B.D. Bartholow, M. Pearson, K.J. Sher, L.C. Wieman, M. Fabiani and G. Gratton, "Effects of Alcohol Consumption and Alcohol Susceptibility on Cognition: A Psychophysiological Examination." Biological Psychology, vol. 64, pp. 167-190, 2003. 
[2] P. Pearson, L.A. Dawe, B. Timney, "Frequency Selective Effects of Alcohol on Auditory Detection and Frequency Discrimination Thresholds." Alcohol and Alcoholism, vol. 34, issue 5, pp. 741-749, 1999.

[3] R. Näätänen, A.W. Gaillard, and S Mäntysalo, "Early selective-attention effect on evoked potential reinterpreted.," Acta Psychol., vol. 42, pp. 313-329, 1978.

[4] T. Koenig and D. Lehmann, "Microstate in language-related brain potential maps show noun-verb differences," Brain Lang, vol. 53, pp. 169-182, 1996.

[5] D. Lehmann and W. Skrandies, "Reference-free identification of components of checkerboard-evoked multichannel potential fields," Electroencephalogr. Cli. Neurophysiol., vol. 48, pp. 609-621, 1980.

[6] D. Lehmann and W. Skrandies, "Spatial analysis of evoked potentials in man: A review," Prog. Neurobiol., vol. 23, pp. 227-250, 1984.

[7] C.M. Michel, M.M. Murray, G. Lantz, S. Gonzalez, L. Spinelli and R. Grave de Peralte, "EEG source imaging," Clin. Neurophysiol., vol. 115, pp. 2195-2222, 2004.

[8] W.K. Strik, T. Dierks, E. Franzek, G. Stöber and K. Maurer, "P300 asymmetries in schizophrenia revisited with reference-independent methods," Psychiatry Res., vol. 55, pp. 153-166, 1994.

[9] J. Wackermann, D. Lehmann, C.M. Michel and W.K. Strik, "Adaptive segmentation of spontaneous EEG map series into spatially defined microstates," Int. J. Psychophys., vol. 14, pp. 269-283, 1993.

[10] D. Lehmann and W. Skrandies, "Segmentation of evoked potentials based on spatial field configuration in multichannel recordings," In W.C. McCallum, R. Zappoli and F. Denoth (eds.) Cerebral Psychophysiology: Studies in Event-Related Potentials. Electroencephalography and Clinical Neurophysiology, Suppl.38., pp. 506-512, 1986.
[11] R.D. Pascual-Marqui, C.M. Michel and D. Lehmann, "Low resolution electromagnetic tomography: a new method for localizing electrical activity in the brain," Int. J. Psychophysiol. vol. 18, pp. 49-65, 1994.

[12] E. Donchin and M.G.H. Coles, "Is the P300 component a manifestation of context updating?," Braib Behav. Sci., vol. 11, pp. 357-374, 1988.

[13] E. Halgren, P. Baudena, J.M. Clarke, G. Heit, C. Liègeois, P. Chauvel and A. Musolino, "Intracerebral potentials to rare target and distractor auditory and visual stimuli. I. Superior temporal plane and parietal lobe," Electroencephalogr. Clin. Neurophysiol., vol. 95, pp. 191-220, 1995.

[14] R. Johnson, "The amplitude of the P300 component of the event related potentials: review and synthesis," In: P.K. Ackles, J.R. Jennings, M.G.H. Coles (Eds.), Advances in Psychophysiology, vol. 2, JAI Press, Greenwich, CT, pp.69-137, 1988.

[15] J. Katayama and J. Polich, "Stimulus context determines P3a and P3b," Psychophysiology, vol. 35, pp. 22-23, 1998.

[16] M. Molnár, "On the origin of the P300 component of the event-related potential component: review and synthesis," Int. J. Psychophysiol., vol. 17, pp. 129-144, 1994.

[17] T.W. Picton, "The P300 wave of the human event-related potential," J. Clin. Neurophysiol., vol. 59, pp. 456-479, 1992.

[18] B. Opitz, A. Mecklinger, A.D. Frederici and D.Y. von Cramon, "The functional neuroanatomy of novelty processing: integrating ERP and fMRI results," Cereb. Cortex, vol. 9, pp. 279-391, 1999.

[19] U. Volpe, A. Mucci, P. Bucci, E. Merlotti, S. Galderisi and M. Maj, "The cortical geneators of P3a and P3b: A LORETA study," Brain Res. Bull., vol. 73, pp. 220-230, 2007. 